\title{
2D chaotic flow in competitive exothermic-endothermic reactions
}

\author{
Z. Huang ${ }^{\text {a }}, \underline{\text { S.D. Watt }}{ }^{\text {a }}$, H. S. Sidhu ${ }^{\text {a }}$, A.C. McIntosh ${ }^{\text {b }}$, J. Brindley ${ }^{\text {c }}$, Z. Jovanoski ${ }^{\text {a }}$ and I.N. Towers ${ }^{\text {a }}$ \\ ${ }^{\text {a }}$ School of Science, UNSW Canberra, Canberra ACT 2600, Australia. \\ ${ }^{b}$ School of Chemical and Process Engineering, University of Leeds, Leeds, LS2 9JT, United Kingdom. \\ ${ }^{c}$ Department of Mathematics, University of Leeds, Leeds, LS2 9JT, United Kingdom. \\ Email: simon.watt@adfa.edu.au
}

\begin{abstract}
We study the effects of chaotic advection in a two-dimensional competitive reaction with an exother-mic reaction and an endothermic reaction and investigate the formation of the filament structure. In a chemical reaction, there are many processes and steps, even in the simplest reactions. By "lumping" these reactions together, a chemical reaction can be modelled using a fewer number of steps. As with any modelling exercise, the more steps that are included, the more complex the model is in terms of the number of system parameters, but this also allows a rich array of behaviour. The model considered here has two reactions, competing for the same fuel source, an exothermic reaction, which generates energy, and an endothermic reaction, which absorbs energy. An example of this type of reaction is the burning of ammonium nitrate in the context of emulsion explosives [Sinditskii et al., 2005].

In one dimension, these exothermic-endothermic reaction models can display complex behaviour. With a suitable initial temperature profile, a travelling r eaction $w$ ave quickly d evelops, $w$ ith $t$ hree $r$ egions: ahead of the reaction where the fuel is unburnt and is at an ambient temperature, behind the reaction where the fuel has been consumed and is at a final burnt temperature and at the reaction front where the fuel is being consumed. Tracking the location of the reaction front, the system can exhibit a range of behaviour. Depending on the system parameters, the speed of the travelling wave can become constant, become oscillatory, with the possibility of period doubling behaviour, or the reaction does not propagate, leading to quenching. This was studied in detail by Sharples et al. [2012]. In two dimensions, similar behaviour can be found [Watt et al., 2019a].

By adding a mixing process to the combustion process, the system behaviour changes. The mixing added to the system was a blinking vortex flow. This flow models the outflow from a large bathtub with with two sinks that are opened in an alternating manner. This alternating flow has been shown to induce chaotic mixing [Károlyi and Tél, 1997]. Kiss et al. [2004] studied this flow as applied to a single step combustion process. It was shown that there is a critical mixing rate, above which the flame is quenched and the reaction s tops. We extend this work by replacing the single step reaction with a two-step competitive reaction. As before, there is a critical mixing rate, above which the flame is quenched. In addition, we explore the sensitivity of the system parameters on the performance of the reaction, as measured by the average steady state temperature.
\end{abstract}

Keywords: Combustion, chaotic mixing, quenching, laminar fluid flow, exothermic 


\section{INTRODUCTION}

We explore the mixing effects of chaotic advection in a fluid on the behaviour of combustion fronts driven by exothermic and endothermic reactions competing for the same fuel. Important practical examples of chaotic advection range from the biological functioning of living organisms, through many fields of science and engineering, to our physical environment as influenced by the geophysical fluid [Neufeld, 2001; Neufeld et al. 2002; Sinditskii et al., 2005; Tel et al., 2005].

We extend a number of previous works. The study of combustion models with chaotic mixing was carried out by Kiss et al. [2004], where the chemical reaction was assumed to be a single-step model. In chemical reaction systems, there are many reactions which occur. However, a commonly used simplification is to "lump" many of these reactions into a smaller number of bulk reactions. However in many practical situations it is not adequate to lump the chemistry into a single reaction model, and it is necessary to retain at least two reactions in the lumped model, either both exothermic or one exothermic and one endothermic. Practical examples are dominated by solid state pyrolysis processes, but liquid cases are exemplified by behaviour of emulsion explosives [Manelis et al. 2003; Sinditskii et al., 2005; Turcotte et al., 2010]. The one-dimensional systems with an exothermic and endothermic reaction without mixing has been studied by Sharples et al. [2012|. More recently, Watt et al. [2019b] studied the reaction with the inclusion of chaotic advection in the one-dimensional model. We extend the system by changing the advection term from a one-dimensional laminar flow to a twodimensional vortex flow.

In Section 2, we develop the chemical reaction model and present the non-dimensionalised governing equations, which is a combination of the non-dimensionalisations discussed in Kiss et al. [2004] and Watt et al. [2019b]. In Section 3, we use an Alternative Direction Implicit method with operator splitting, as discussed in Kiss et al. |2004|, to obtain numerical solutions. Numerical solutions are obtained to explore the behaviour of the system with respect to the system parameters. Using the average temperature as an indicator of behaviour, the sensitivity of the system behaviour to the system parameters is explored in Section 4.

\section{MATHEMATiCAL MODEL}

For this study, we will consider a model of combustion similar to that described in Kiss et al. [2003, 2004|; Watt et al. [2019b], which includes diffusion, reaction and advection terms

$$
\begin{aligned}
\rho c_{p}\left(\frac{\partial T}{\partial t}+\mathbf{v} \cdot \nabla T\right) & =\kappa \Delta T-\rho Q_{1} C K_{1}(T)+\rho Q_{2} C K_{2}(T), \\
\frac{\partial C}{\partial t}+\mathbf{v} \cdot \nabla C & =D \Delta C-C K_{1}(T)-C K_{2}(T)
\end{aligned}
$$

where $T$ and $C$ represent temperature and fuel concentration, respectively, $t$ time, $\rho$ the density, $c_{p}$ the specific heat, $Q_{1}$ and $Q_{2}$ the enthalpies of the endothermic and exothermic reaction, $\kappa$ and $D$ the thermal conductivity and diffusion coefficient, respectively. The terms $K_{1}(T)$ and $K_{2}(T)$ are the reaction rate constants, characterized by highly nonlinear dependence of temperature. $K_{1}(T)$ and $K_{2}(T)$ generally assumed to obey the Arrhenius law can be written as

$$
K_{i}(T)= \begin{cases}0, & T \leq T_{i}, \\ A_{i} \exp \left(-\frac{E_{i}}{R T}\right), & T>T_{i},\end{cases}
$$

where $i=1$ and $i=2$ denote the endothermic and exothermic reactions, respectively, $T_{i}, A_{i}$ and $E_{i}$ are the ignition temperature, pre-exponential factors and the activation energies, respectively, and $R$ is the universal gas constant. By including an ignition temperature $\left(T_{i}\right)$, the cold boundary problem Zeldovich et al. $|1985|$ is avoided. For simplicity, we set the ignition temperature $T_{1}=T_{2}=T_{a}$, where $T_{a}$ is the ambient temperature.

The velocity field is assumed to be the superposition of a point sink and a point vortex. Following Károlyi and Tél |1997|; Kochin et al. [1963|; Kiss et al. |2003], the complex potential centered at $\left(z_{s}=x_{s}+i y_{s}\right)$ is

$$
w(z)=-(J+i K) \ln \left|z-z_{s}\right|,
$$

where $z=x+i y$ is the complex coordinate in the plane of the flow and $J$ and $K$ represent the sink strength and the vortex strength, respectively. The velocity field corresponding to $w(z)$ consists of the superposition of a radial component $v_{r}=-J / r$ and a tangential component component $v_{\varphi}=K / r$, where 
Z. Huang et al, 2D chaotic flow in competitive exothermic-endothermic reactions

$r=\sqrt{\left(x-x_{s}\right)^{2}+\left(y-y_{s}\right)^{2}}$. The imaginary part of the complex potential, $\Phi=-K \ln r-J \varphi$ is the stream function and the streamlines of the level lines of $\Phi$ are logarithmic spirals $\varphi=-K / J \ln r+$ Constant. In Cartesian coordinates, the velocity field, $\mathbf{v}=\left(v_{x}, v_{y}\right)$, can be written as

$$
v_{x}=\frac{-J\left(x-x_{s}\right)-K\left(y-y_{s}\right)}{r^{2}}, \quad v_{y}=\frac{K\left(x-x_{s}\right)-J\left(y-y_{s}\right)}{r^{2}} .
$$

In line with the analysis carried out in Kiss et al. [2003|, the velocity field is assumed to be a blinking vortexsink system, having two sinking vortex points, one located at $z_{s, 1}=(\ell, 0)$ and which is the other at $z_{s, 2}=$ $(-\ell, 0)$. The flow is assumed to be periodic, with period $T_{0}$, with the vortex at $z_{s, 1}$ being active for the first half of the period and the vortex at $z_{s, 2}$ being active for the second half of the period.

The spatial and temporal domains of the system (1) and (2) are $\Omega=[-L / 2, L / 2] \times[-L / 2, L / 2]$ and $t \geq 0$. This system is subject to the following boundary conditions that

$$
T(\mathbf{r}, t)=T_{a}, \quad C(\mathbf{r}, t)=C_{0} \quad \text { on } \quad \mathbf{r} \in \partial \Omega \quad(t \geq 0) .
$$

The length $L$ is assumed to be large enough to alleviate any edge effects in the numerical solution.

We introduce dimensionless variables

$$
u=\frac{R T}{E_{2}}, \quad c=\frac{C}{C_{0}}, \quad \mathbf{r}^{\prime}=\frac{\mathbf{r}}{\ell}, \quad t^{\prime}=\frac{t}{T_{0}} .
$$

Substituting (7) into the system (1) and (2) and omitting the primes leads to

$$
\begin{aligned}
\frac{\partial u}{\partial t}+v_{x} \frac{\partial u}{\partial x}+v_{y} \frac{\partial u}{\partial y} & =-\frac{q r \operatorname{Da}}{\Theta} c k_{1}(u)+\frac{\mathrm{Da}}{\Theta} c k_{2}(u)+\operatorname{Pe}^{-1} \operatorname{Le} \Delta u \\
\frac{\partial c}{\partial t}+v_{x} \frac{\partial c}{\partial x}+v_{y} \frac{\partial c}{\partial y} & =-r \operatorname{Da} c k_{1}(u)-\operatorname{Da}_{c} k_{2}(u)+\mathrm{Pe}^{-1} \Delta c
\end{aligned}
$$

where

$$
\begin{gathered}
v_{x}=\frac{-\eta\left(x-x_{s}\right)-\eta \xi\left(y-y_{s}\right)}{\left\|\mathbf{r}-\mathbf{r}_{\mathbf{s}}\right\|^{2}}, \quad v_{y}=\frac{\eta \xi\left(x-x_{s}\right)-\eta\left(y-y_{s}\right)}{\left\|\mathbf{r}-\mathbf{r}_{\mathbf{s}}\right\|^{2}} \\
\mathrm{Pe}=\frac{\ell^{2}}{D T_{0}}, \quad \mathrm{Da}=A_{2} T_{0}, \quad \eta=\frac{J T_{0}}{\ell^{2}}, \quad \xi=\frac{K}{J}, \\
L e=\frac{\kappa}{\rho c_{p} D}, \quad \Theta=\frac{c_{p} E_{2}}{C_{0} Q_{2} R}, \quad q=\frac{Q_{1}}{Q_{2}}, \quad r=\frac{A_{1}}{A_{2}} .
\end{gathered}
$$

We refer to the parameter Pe as the Péclet number, Da as the Damköhler number, Le as the Lewis number, $\Theta$ as the exothermicity parameter, $q$ as the ratio of enthalpies and $r$ as the ratio of reaction constants. It should be noted that by setting $q=0$ (which corresponds to $Q_{1}=0$ ), the system reduces to the one-step exothermic system as studied by Kiss et al. [2004]. However, a different dimensionless temperature was used and thus the results are not directly comparable.

The temperatures dependence of the reaction (3) become

$$
k_{1}(u)=\left\{\begin{array}{ll}
0, & u \leq u_{a} \\
\exp \left(-\frac{f}{u}\right), & u>u_{a}
\end{array}, \quad k_{2}(u)=\left\{\begin{array}{ll}
0, & u \leq u_{a} \\
\exp \left(-\frac{1}{u}\right), & u>u_{a}
\end{array},\right.\right.
$$

where

$$
u_{a}=\frac{R T_{a}}{E_{2}}, \quad f=\frac{E_{1}}{E_{2}},
$$

with $u_{a}$ the dimensionless ambient temperature and $f$ is the ratio of activation energies. The corresponding boundary conditions in dimensionless variables are

$$
u(\mathbf{r}, t)=u_{a}, \quad c(\mathbf{r}, t)=1 \quad \text { on } \quad \mathbf{r} \in \partial \Omega \quad(t \geq 0) .
$$

The Damköhler number, Da, characterizes the ratio between the advective and the exothermic reaction timescales. Large Damköhler number corresponds to slow stirring (large time period $T_{0}$ ) or equivalently fast exothermic reaction (large pre-exponential factor $A_{2}$ ) and vice versa. The Péclet number, Pe, is a measure of the relative strength of advective and diffusive transport. 


\section{NUMERicAl RESULTS}

\subsection{Method}

The reaction-advection-diffusion problem (8), (9) and (14) is solved using the Alternative Direction Implicit method for the advection-diffusion terms and a Runge-Kutta solver for the reaction terms, as outlined in Kiss et al. |2004|. The domain is discretised into a thousand steps in each direction and the length of the domain is assumed to be ten units, giving a grid spacing of $\delta x=\delta y=0.01$. The time steps are $\delta t=0.001$ and the simulations are run for sixty time units.

The performance metrics will be the average dimensless temperature and fuel consumption, defined as

$$
\bar{u}=\frac{1}{L^{2}} \int_{\Omega} u(x, y, t) d x d y, \quad \bar{c}=\frac{1}{L^{2}} \int_{\Omega}[1-c(x, y, t)] d x d y
$$

As with the one-dimensional problem, as studied by Watt et al. [2019b], there is a critical Damköhler number, below which the combustion process does not initiate and the temperature is at ambient temperature over the domain. This corresponds to the regime where there is fast stirring and the flame is quenched. For Damköhler numbers above the critical value, the combustion does initiate and a flame filament occurs.

\subsection{Results}

As we are interested in strongly advective flow, we consider large Péclet numbers and set it as Pe $=2000$ for the rest of the paper, as given in [Kiss et al., 2004]. For all of the following simulations, the initial fuel load will be unconsumed $(c=1)$ and the temperature profile is set at ambient temperature plus a small Gaussian perturbation at the origin as follows

$$
u(x, y, 0)=u_{a}+A e^{-b\left(x^{2}+y^{2}\right)} .
$$

For the numerical results presented, we set $u_{a}=0, A=1$ and $b=4$. In Figure 1, we give a sequence of temperature plots for $\mathrm{Le}=1, \mathrm{Da}=10, \eta=0.1$ and $\xi=20$. The yellow colour corresponds to the higher temperatures, while the darker blue colour corresponds to the lower temperatures. The sequence starts at $t=0$ with the sink located at $(1,0)$ opened. The sink point switches to $(-1,0)$ when $0.5<t \leq 1$. The spiral temperature filaments are first formed around the vortex-sink point and then the temperature filaments would eventually converge to the steady periodic state, as seen in Figure 1 for $t \geq 9$. Regions of relatively high temperatures are located at the open sink. It is clear that there exist two vortex-sink points. Similar results can be found in hydrodynamic flows Károlyi and Tél |1997|. The temperature plots for Le $=0.1$ is presented in Figure 2. The temperature filament structures for $\mathrm{Le}=0.1$ and $\mathrm{Le}=1$ are similar. However, the corresponding temperatures are slightly higher for $\mathrm{Le}=0.1$, compared to $\mathrm{Le}=1$.

In Figure 3, we consider different values of flow parameters, $\eta$ and $\xi$. The steady state temperature profile are similar to the flows found in Figure 10 of Károlyi and Tél |1997|. This suggest that the advection is the dominant factor with regards to the shape of the reaction zone.

We will be focusing on intermediate values of the Damköhler number. Large Damköhler numbers corresponds to small strain rates. As the Damköhler number approaches infinity, the strain rate approaches zero and the system reduces to an endothermic-exothermic combustion reaction without mixing (see for example Sharples et al. |2012 | for the one-dimensional system and Watt et al. |2019a| for the two-dimensional system). Similarly, small Damköhler numbers correspond to large strain rates and the flame is quenched. An example of this critical Damköhler number is presented in Figure 4. For a Damköhler number above the critical value, a flame filament develops and for a Damköhler number below the critical value, the flame is quenched. The dependence of the critical Damköhler number on the system parameters is beyond the scope of this paper, but will be explored in future work.

\section{SENSITIVITY OF SYSTEM PARAMETERS ON THE PERFORMANCE}

In the system outlined in Section 2, there are nine parameters which affect the behaviour and performance of the system. Of these, two correspond to the diffusion terms (Pe and Le), four correspond to the reaction terms $(q, r, f$ and $\Theta$ ) and three correspond to the advection terms (Da, $\eta$ and $\xi$ ). The Péclet number is fixed at $\mathrm{Pe}=2000$, but the remainder of the parameters can be varied. We undertake a preliminary exploration of the variation of these parameters on the dynamics of the system. 

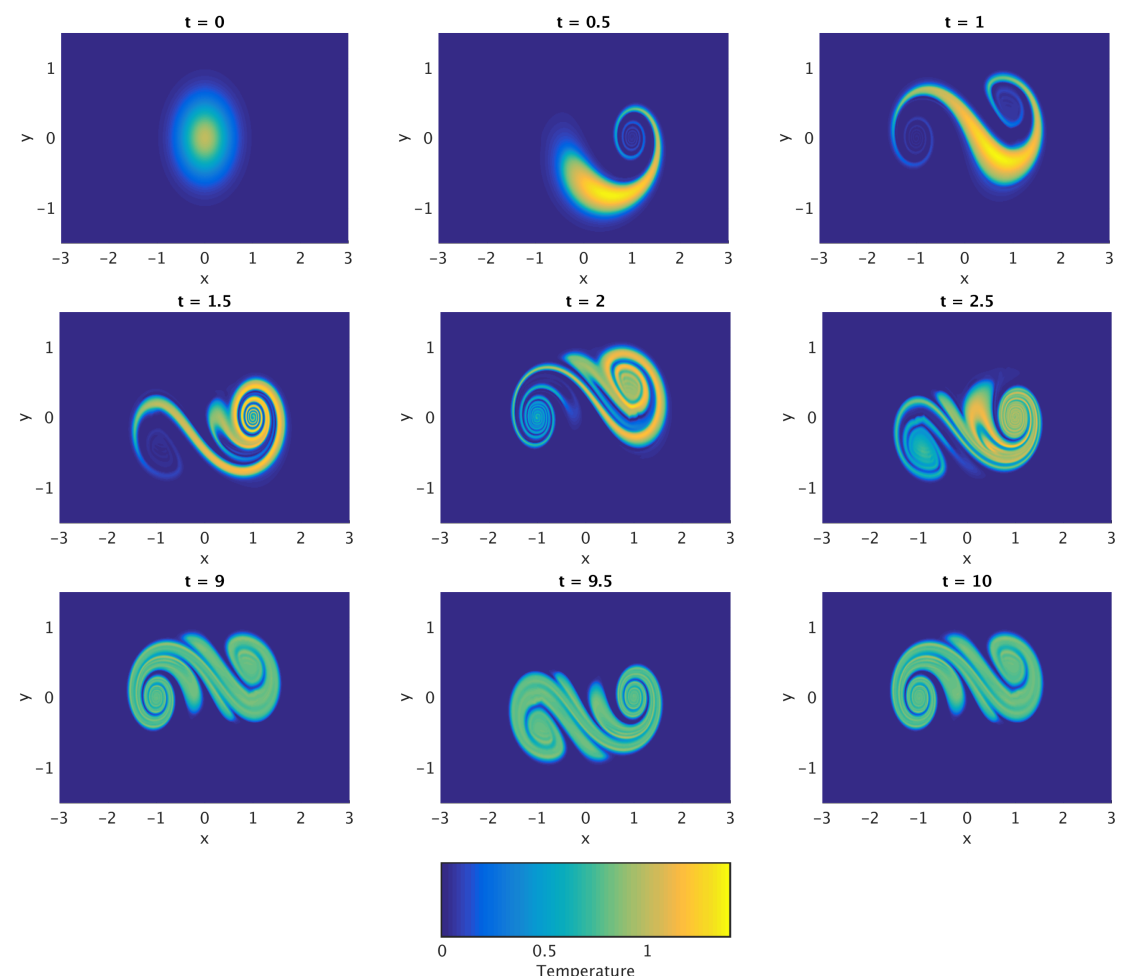

Figure 1. Temperature profiles with $q=1, r=1, f=2, \Theta=1, \mathrm{Le}=1$, Da $=10$, $\eta=0.1, \xi=20$ at times shown.
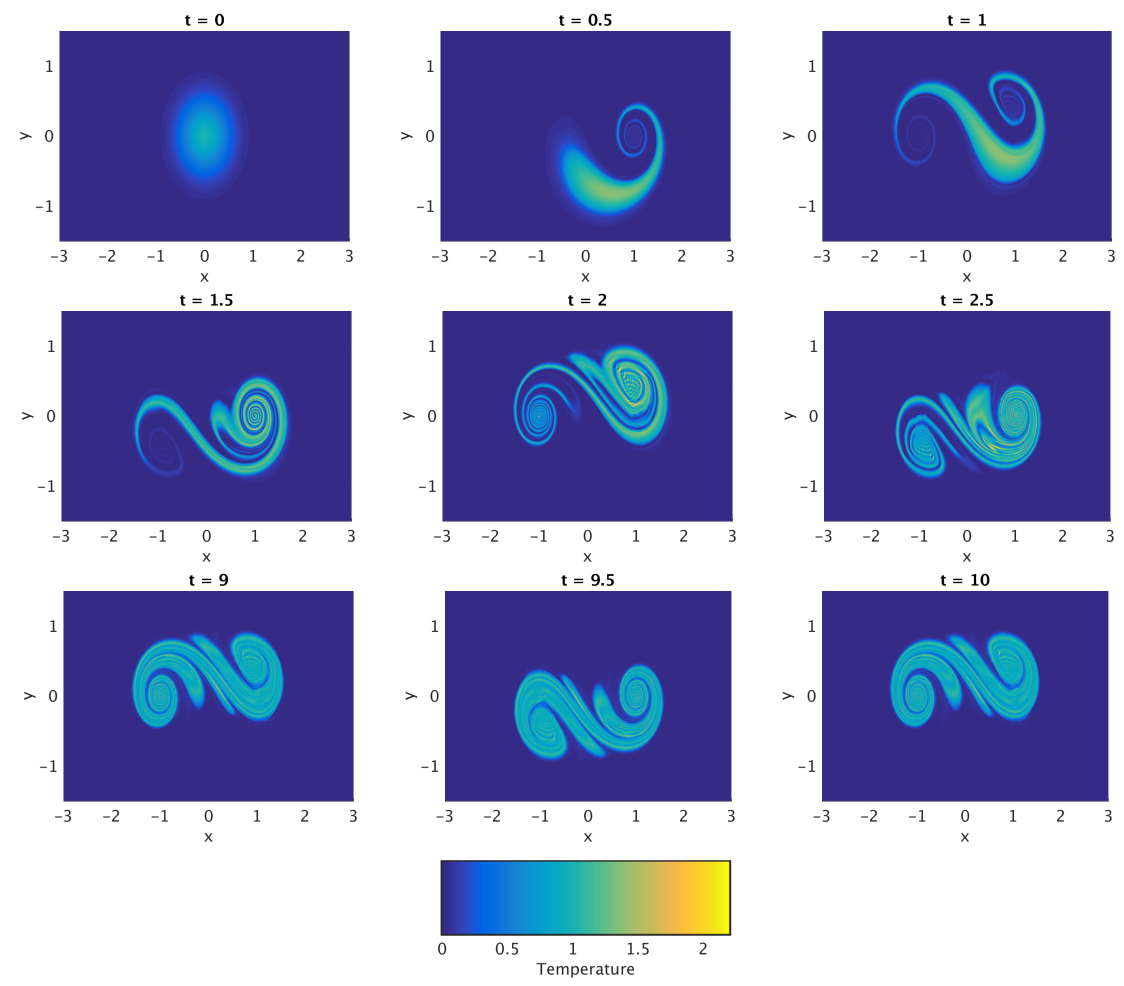

Figure 2. Temperature profiles with $q=1, r=1, f=2, \Theta=1$, $\mathrm{Le}=0.1$, Da $=$

$$
10, \eta=0.1, \xi=20 \text { at times shown. }
$$


Z. Huang et al, 2D chaotic flow in competitive exothermic-endothermic reactions
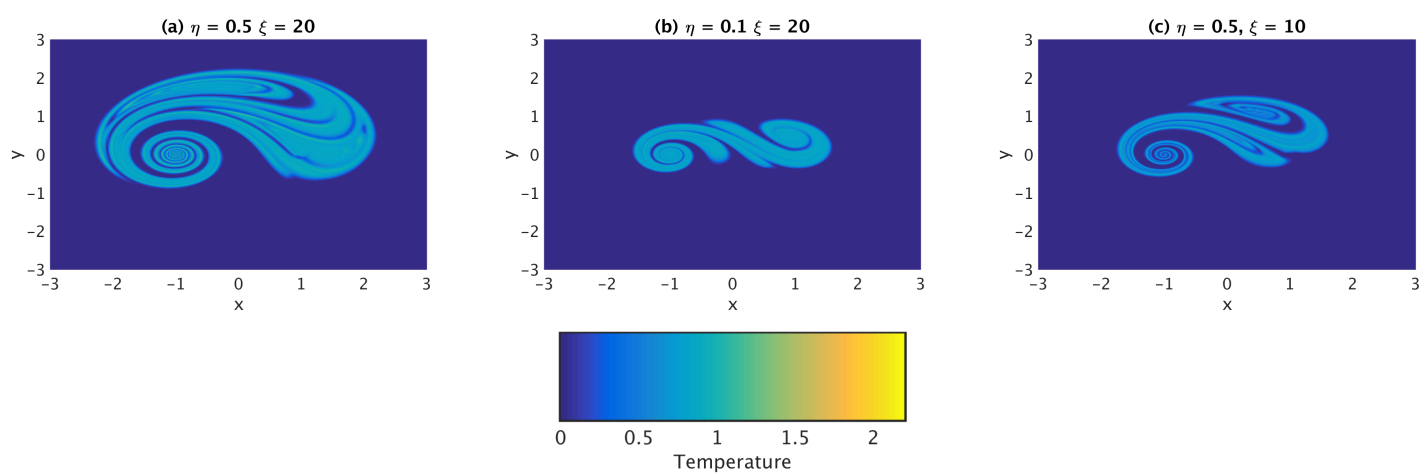

Figure 3. Temperature filaments with $q=1, r=1, f=2, \Theta=1$, Le $=0.1$, Da $=10$ (a) $\eta=0.5$ and $\xi=20$ (b) $\eta=0.1$ and $\xi=20$ and (c) $\eta=0.5$ and $\xi=10$.
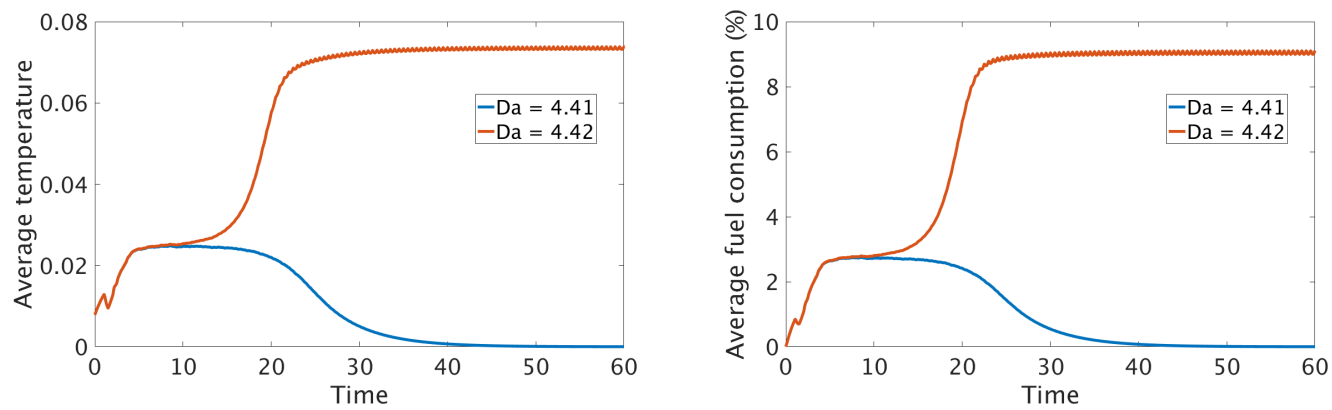

Figure 4. Average temperature and average fuel consumption with $q=1, r=1, f=2, \Theta=1$, Le $=0.1, \eta=$ 0.1 and $\xi=20$ for one Damköhler number below $(\mathrm{Da}=4.41)$ and one above $(\mathrm{Da}=4.42)$ the critical value.

To study the effect of varying each of these parameters, we perturb about the base case, as given in Figure 3a, by adjusting each value above or below the base value by ten percent. The results are shown in Table 1 . From the results, we can see that ratio of the vortex to sink strength, $\xi$, has the largest effect on the dynamics. In addition, the ratio of activation energies, $f$, sink strength, $\eta$ and exothermicity, $\Theta$, also has marked effects on the behaviour of the system.

\section{Conclusions}

We undertook a preliminary investigation of chaotic mixing in combustion flows with two competitive reactions, one exothermic and one endothermic. The application of such flows can be seen in areas such as biological functioning of living organisms and geophysical fluids. The model presented here is an extension to the model of combustion with an exothermic reaction and an endothermic reaction, with no mixing, and a single-step model of combustion with mixing.

In this work, we explored the sensitivity of the system behaviour with respect to the the various model parameters, which described the mixing, diffusion and reaction mechanisms. We found that ratio of the vortex to sink strength had the largest effect on the dynamics of the system, as measured by the average temperature. The vortex strength, exothermicity and the ratio of activation energies also had a marked effect on the dynamics.

Future work will include a more detailed exploration of the system parameters. As mentioned previously, the system with chaotic mixing and a single-step reaction has been extensively studied by Kiss et al. [2004]. We do not wish to replicate these results, but to explore the difference which arise from extending the model to a competitive exothermic-endothermic reaction system. 
Z. Huang et al, 2D chaotic flow in competitive exothermic-endothermic reactions

Table 1. Summary of the effect the variation of the system parameters has on the performance indicator, as measured by the average temperature, $\bar{u}$.

\begin{tabular}{|c|c|c||}
\hline $\begin{array}{c}\text { Parameter change } \\
\text { from base case }\end{array}$ & $\begin{array}{c}\text { Performance indicator } \\
\bar{u}\end{array}$ & Change in indicator (\%) \\
\hline \hline Base case & 0.0588 & - \\
\hline $\mathrm{Le}=0.9$ & 0.0596 & 1.4 \\
$\mathrm{Le}=1.1$ & 0.0581 & -1.3 \\
\hline $\mathrm{Da}=9$ & 0.0560 & -4.8 \\
$\mathrm{Da}=11$ & 0.0610 & 3.8 \\
\hline$\eta=0.45$ & 0.0526 & -10.5 \\
$\eta=0.55$ & 0.0650 & 10.6 \\
\hline$\xi=18$ & 0.0480 & -18.4 \\
$\xi=22$ & 0.0703 & 19.6 \\
\hline$\Theta=0.9$ & 0.0658 & 12.0 \\
$\Theta=1.1$ & 0.0521 & -11.4 \\
\hline$q=0.9$ & 0.0605 & 2.9 \\
$q=1.1$ & 0.0572 & -2.8 \\
\hline$r=0.9$ & 0.0612 & 4.2 \\
$r=1.1$ & 0.0565 & -3.9 \\
\hline$f=1.8$ & 0.0504 & -14.3 \\
$f=2.2$ & 0.0652 & 11.0 \\
\hline
\end{tabular}

\section{REFERENCES}

I.Z. Kiss, J.H. Merkin, S.K. Scott, P.L. Simon, S. Kalliadasis, and Z. Neufeld. The structure of flame filaments in chaotic flows. Physica D: Nonlinear Phenomena, 176(1):67-81, 2003. doi: https://doi.org/10.1016/ S0167-2789(02)00741-8.

I.Z. Kiss, J.H. Merkin, and Z. Neufeld. Homogenization induced by chaotic mixing and diffusion in an oscillatory chemical reaction. Physical Review E, 70:026216-1-026216-11, 2004.

N.E. Kochin, I.A. Kibel, and N.V. Rose. Theoretical Hydrodynamics. Interscience, New York, 1963.

G. Károlyi and T. Tél. Chaotic tracer scattering and fractal basin boundaries in a blinking vortex-sink system. Physics Reports, 290(1):125 - 147, 1997. ISSN 0370-1573. doi: https://doi.org/10.1016/S0370-1573(97) $00063-\mathrm{X}$.

G. B. Manelis, G.M. Nazin, Yu. I. Rubtsov, and V. A. Strunin. Thermal Decomposition and Combustion, pages 175-187. Taylor and Francis, London, 2003.

Z. Neufeld. Excitable media in a chaotic flow. Phys. Rev. Lett., 87:108301, 2001.

Z. Neufeld, P. H. Haynes, V. Garcon, and J. Sudre. Ocean fertilization experiments may initiate large scale phytoplankton bloom. Geophys. Res. Lett., 29:1534-1537, 2002.

JJ Sharples, HS Sidhu, AC McIntosh, J Brindley, and VV Gubernov. Analysis of combustion waves arising in the presence of a competitive endothermic reaction. IMA Journal of Applied Mathematics, 77(1):18-31, 2012.

V. P. Sinditskii, V. Yu Egorshev, A. I. Levshenkov, and V. V. Serushkin. Ammonium nitrate: combustion mechanism and the role of additives. Propellants, Explosives andPyrotechnics, 30(4):269-280, 2005.

T. Tel, A. de Moura, C. Grebogi, and G. Karolyi. Chemical and biological activity in open flows : a dynamical systems approach. Phys Reports - Review section of Physics Letters, 413:91-96, 2005.

R. Turcotte, S. Goldthorpe, C. M. Badeem, H. Feng, and S. K. Chan. Influence of physical characteristics and ingredients on the minimum burning pressure of ammonium nitrate emulsions. Propellants, Explosives andPyrotechnics, 35(3):233-239, 2010.

S.D. Watt, Z. Huang, and H.S. Sidhu. Analysis of two-dimensional combustion waves arising in the presence of a competitive endothermic reaction. ANZIAM Journal, 2019a.

S.D. Watt, Z. Huang, H.S. Sidhu, A.C. McIntosh, and J. Bridley. One dimensional chaotic laminar flow with competitive exothermic-endothermic reactions. ANZIAM Journal, $2019 \mathrm{~b}$.

Y. B. Zeldovich, G. I. Barenblatt, V. B. Librovich, and G. M. Makhviladze. The Mathematical Theory of Combustion and Explosions. Springer, New York : Consultants Bureau, 1985. 